Results Between 2010-2018, 5681 syphilis cases were diagnosed in BC, including $61(1.1 \%)$ diagnosed with OS. The proportion of syphilis cases with OS increased from $0.48 \%$ in 2010 to $2.99 \%$ in 2018 ( $\mathrm{P} \leq 0.05)$. Among OS cases, median age was 47 years, $88.5 \%$ were male and the most common ophthalmologic diagnosis was panuveitis (44.3\%). At the time of syphilis diagnosis, $50.8 \%$ of OS cases were HIV-positive compared to $25.8 \%$ of controls $(\mathrm{P} \leq 0.001)$. Among individuals with HIV co-infection, OS cases had higher viral loads (42.9\% vs $79.7 \%$ were suppressed; $\mathrm{P} \leq 0.001)$ and lower median CD4 counts (470 vs 615 cells $/ \mathrm{mm}^{3} ; \mathrm{P} \leq 0.05$ ) than controls. Factors associated with OS were primary/secondary stage of syphilis (adjusted odds ratio [aOR] 4.06; 95\% confidence interval $[\mathrm{CI}]$ 1.52-10.8), early latent stage of syphilis (aOR 3.71; 95\%CI 1.39-9.95), and HIV-positive serostatus (aOR $2.49 ; 95 \%$ CI $1.27-4.88)$.

Conclusion OS incidence and proportion increased over the study period in BC. These findings highlight the importance of vigilance for OS, particularly those in the early stages of syphilis and those living with HIV, to avoid diagnostic and treatment delays.

Disclosure No significant relationships.

\section{P124 ATTITUDES AND BELIEFS REGARDING HIV AND AIDS AMONG IMMIGRANTS IN MOPANI DISTRICT, SOUTH AFRICA}

Hilda Shilubane* „_unic Khoza. University of Venda, Thohoyandou, South Africa

\subsection{6/sextrans-2019-sti.301}

Background Sub-Saharan Africa faces and is severely affected by many conflicts. Human Immunodeficiency Virus (HIV) and Acquired Immunodeficiency Syndrome (AIDS) threaten both the physical and financial well-being of individuals in struggling countries. Research indicates that negative attitude towards HIV/AIDS as well as persons infected with the virus persist despite the increased knowledge of prevention. This research aims to explore and describe the immigrants' attitudes and beliefs regarding HIV and AIDS in the Mopani district, Limpopo Province.

Methods A quantitative descriptive design was used. The target population was all immigrants at Mopani district in Limpopo province and the sample comprised of males and females. Data was collected using a questionnaire with closed and open-ended questions. Ethical standards were maintained.

Results The study revealed that many participants expressed discriminatory practice towards individuals infected by HIV and those with AIDS. Many viewed promiscuity and the disease called Makhume (meaning illness caused by the omission of purification rites following the death of a family member) as the cause for HIV and AIDS.

Conclusion The findings may hinder the achievement of healthy lives and promotion of well-being at all ages if not addressed appropriately. The collaboration of various departments (Health, Social development, Education, and etc.) in the Mopani district is required to change these negative attitudes and beliefs that influence immigrants' behaviour. In addition, the use of audio-visuals and peer teaching may help to change their attitudes since they have shown to be most successful in changing the attitudes.

Disclosure No significant relationships.

\section{P125 LOW RATE OF HIV TESTING AMONG PRISONERS IN IRAN: FINDINGS FROM NATIONWIDE REPEATED BEHAVIORAL SURVEYS}

${ }^{1}$ Mohammad Karamouzian*, ${ }^{2}$ Najmeh Parhizgari, ${ }^{3}$ Mostafa Shokoohi, ${ }^{3}$ Fatemeh Tavakoli, ${ }^{3}$ Armita Shahesmaeili, ${ }^{4}$ Ali Mirzazadeh, ${ }^{3}$ Hamid Sharifi. ${ }^{1} B C$ Center on Substance Use, Vancouver, Canada; ${ }^{2}$ Tehran University of Medical Sciences, Tehran, Iran; ${ }^{3}$ WHO Collaborating Center for HIVISTI Surveillance, KERMAN, Iran; ${ }^{4}$ UCSF, San Francisco, USA

\subsection{6/sextrans-2019-sti.302}

Background Prisoners are disproportionately at increased risk of HIV infection. Despite the importance of assessing HIV testing rates among key populations at risk of HIV, our understanding of HIV testing practices of Iranian prisoners is limited. This study utilizes data from two repeated behavioral survey studies to shed light on the prevalence and correlates of HIV testing among Iranian prisoners.

Methods Data were obtained from two consecutive surveys in $2009(\mathrm{~N}=5913)$ and $2013(\mathrm{~N}=5430)$ using a multistage random sampling approach including both large and small prison settings. Having a recent HIV test result was defined as having an HIV test result over the last 12 months. Biological and survey data were collected through dried blood spot testing and standardized risk assessment questionnaire, respectively. Descriptive statistics including frequencies, percentages, and $95 \%$ confidence intervals (CI) were reported. Bivariable and multivariable logistic regression models were built to compare the odds of having a recent HIV test result among different subgroups of prisoners.

Results Most participants had never tested for HIV $(68.7 \%$ in 2009 and $62.2 \%$ in 2013). Only 9.9\% (in 2009) and $11.6 \%$ (in 2013) of the participants had a recent HIV test result. Older age $(\mathrm{AOR}=1.50)$, lifetime history of drug use $(\mathrm{AOR}=1.87)$ and sexual intercourse $(\mathrm{AOR}=1.42)$, and self-perceived risk of HIV $(\mathrm{AOR}=0.74)$ were significantly associated with recent HIV testing in 2009. Female sex $(\mathrm{AOR}=4.03)$, multiple incarcerations $(\mathrm{AOR}=1.40)$, alcohol consumption $(\mathrm{AOR}=1.23)$, and STI $(\mathrm{AOR}=1.79)$ and HIV knowledge $(\mathrm{AOR}=1.62)$ were significant correlates in 2013 .

Conclusion HIV testing is extremely low among prisoners in Iran. There is an urgent need to revisit current HIV testing policies across Iranian prisons and identify the barriers to HIV testing within the prison settings. Scaling up rapid tests as well as routine opt-out HIV testing services could help encourage prisoners to further use the available harm reduction facilities inside prisons.

Disclosure No significant relationships.

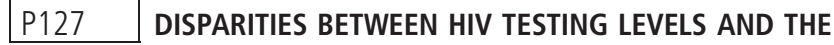 SELF-REPORTED HIV-NEGATIVE STATUS OF SEXUALLY ACTIVE COLLEGE STUDENTS}

Edmond Pui Hang Choi ${ }^{1 *}$, Janet Yuen Ha Wong ${ }^{2}$, Daniel Yee Tak Fong ${ }^{1}{ }^{1}$ The University of Hong Kong, School of Nursing, Hong Kong, PRC; ${ }^{2}$ The University of Hong Kong, PRC

\subsection{6/sextrans-2019-sti.303}

Background Despite the availability of rapid point-of-care human immunodeficiency virus (HIV) testing, the use of a self-reported HIV status has its own value, especially in research studies. Sexually active people without prior HIV testing should report as 'HIV status unknown' or 'never tested' instead of 'HIV negative' when they answer the question item on HIV status. The study aimed to assess the 
prevalence of HIV testing and the disparities between HIV testing levels and self-reported HIV status in college students in Hong Kong.

Methods College students were recruited in four Hong Kong universities. Recruited subjects completed a structured questionnaire about their sexual behaviours. The prevalence of HIV testing and the inaccurate disclosure of HIV status (reporting an HIV-negative status without prior HIV testing) was estimated in sexually active students, and men who have sex with men (MSM) students, respectively.

Results Two hundred and fifty five students were included in the analysis. $16.1 \%$ of subjects were MSM. Overall, $81.6 \%$ of subjects reported that they had never had any HIV testing. $65.9 \%$ of subjects had inaccurate self-disclosure. Among subjects who had inconsistent condom use, only $20.2 \%$ of the subjects had HIV testing and $66.7 \%$ of the subjects had inaccurate self-disclosure. Among MSM students, only 36.6\% of the subjects had HIV testing before and $61.0 \%$ had inaccurate self-disclosure. Among MSM student who had inconsistent condom use, only $35.1 \%$ of the subjects had HIV testing and $62.2 \%$ had inaccurate self-disclosure.

Conclusion It was alarming to find the low prevalence of HIV testing but the high prevalence of inaccurate HIV status disclosure among sexually active college students, especially among MSM. The study findings raised the methodological issue of using a self-reported HIV status. Biological testing should be used whenever feasible, and public health interventions to promote HIV testing should be continued.

Disclosure No significant relationships.

\section{P128 HIV+ WOMEN 50+ STIGMATIZED AND DISCRIMINATED}

Chantal Mukandoli*. APAA (African In Partnership Against AIDS), Women Support, Toronto, Canada

10.1136/sextrans-2019-sti.304

Background Women living with HIV 50+years and older are facing, stigma while disclosing their status to their family friends, community, and partners. Women living with HIV are empowering and educating each other. Women face different issues (stress, depression, isolation, trauma or mental health issues). Disclosure of HIV status present challenges, there is need to support around women how to disclose and when. Women also are at higher risk of being criminalized by law for no-disclosure.

Methods We created social group in safe places for women living with HIV to come together to learn from each other regarding the issues they face. Women can speak about relationship, medications, health issues, and accessing services and program. Build each other's capacity skills. They gain a sense of connection through cooking, sewing, knitting. Peers who run the group are trained. The sessions were conducted in different sessions in the event. We administered pre- and postsession questionnaire to determine the effectiveness of the sessions. Demographic of those attending the group $87 \%$ identify as female, $13 \%$ identify as transwomen. Age $45-50+28 \%$, $50+72 \%$ attended group session.

Results (50) women attended this group sessions and received information and connected with other women living with HIV. 10 women reported that they have come out of isolation and are now support and mentoring other women in their communities. 5 women have been engaged in advocacy at the community and represent women in various working groups and committees.

Conclusion We are confident that social groups for women living with HIV help women to seek services, remain in care, reduce isolation and promote adherence. Meaningful involvement is more that inviting women at decision making tables but also addressing barriers that hinder women from engaging, addressing stigma, and other personal barriers that women might have.

Disclosure No significant relationships.

\section{P137 MODELLING FACTORS DETERMINING PAKISTAN'S HETEROGENEOUS HIV EPIDEMIC IN PEOPLE WHO INJECT DRUGS}

${ }^{1}$ Aaron Lim*, ${ }^{1}$ Adam Trickey, ${ }^{2}$ Laura Thompson, ${ }^{3}$ Tahira Reza, ${ }^{2}$ Faran Emmanuel, ${ }^{4}$ James Blanchard, ${ }^{1}$ Peter Vickerman. 'University of Bristol, Population Health Sciences, Bristol Medical School, Bristol, UK; ${ }^{2}$ University of Manitoba, Centre for Global Public Health, Department of Community Health Sciences, WINNIPEG, Canada; ${ }^{3}$ Centre for Global Public Health, Islamabad, Pakistan; ${ }^{4}$ University of Manitoba, Centre for Global Public Health Winnipeg, Canada

\subsection{6/sextrans-2019-sti.305}

Background Pakistan's HIV epidemics among people who inject drugs (PWID) vary widely across different cities and has increased dramatically over time. To help guide future HIV programming, we used statistical and mathematical modelling to identify possible causes for these differing HIV epidemics.

Methods Cross-sectional integrated biological and behavioural surveillance (IBBS) data describing high-risk behaviours and HIV status among PWID were collected by the Canada-Pakistan HIV/AIDS Surveillance Project (HASP) over five rounds from 2005-2017. We used regression analyses to identify citylevel associations between the prevalence of HIV infection and different risk behaviours. We then developed a dynamic HIV transmission model to reflect these associations. The model was calibrated to the IBBS dataset to estimate relative risks of HIV transmission due to each risk factor, and their respective population-attributable fractions (PAFs) over 10-years. Lastly, we investigated the prevention benefit that could be achieved if these behaviours were reduced across different settings to the lowest observed values.

Results Multivariable statistical analyses identified professional injecting use at last injection ('ProfInj') and heroin use within the past month ('HeroinUse') as key high-risk behaviours associated with city-level HIV infection, with HIV prevalence increasing by $4 \%$ and $2 \%$ for every $10 \%$ increase in the prevalence of each respective behaviour. Modelling projections estimated that ProfInj and HeroinUse increased the relative risk of HIV transmission by 7.9 (95\%CrI 4.6-14.0) and 2.2 (1.13.7) times, respectively, with 10 -year PAFs across all cities being 52\% (37-61\%) and 21\% (3-34\%), separately, and 61\% (51-66\%) combined. Lowering each behaviour's prevalence across all cities to the lowest observed prevalence (11\% ProfInj, 0\% HeroinUse) reduced overall relative HIV incidence by $43.9 \%(36.9-47.7 \%)$ and $44.1 \%(11.0-60.5 \%)$ over 10 -years, respectively.

Conclusion Pakistan's HIV epidemic is heterogeneous, with professional injecting and heroin use likely large contributors to the differing epidemics. Interventions focussed on these behaviours, especially professional injecting, could substantially reduce HIV incidence.

Disclosure No significant relationships. 